\title{
Mapeamento Sistemático: Museu em Realidade Aumentada
}

\author{
Luan M. G. de Oliveira, Luciana de Oliveira Berretta, Taciana Novo Kudo \\ Instituto de Informática - Universidade Federal de Goiás(UFG) \\ Goiânia - GO - Brazil \\ luan.macedo@discente.ufg.br, (luciana.berretta, taciana)@ufg.br
}

\begin{abstract}
Augmented Reality (AR) presents highly adaptive and evolutionary behaviors and great potential in cultural heritage for generating more attractive and immersive environments in audiovisual aspects. In this sense, this work carries out a secondary study in the form of Systematic Mapping, which identifies and analyzes primary studies on Virtual Museum proposals developed using AR techniques for education. We raised some of the advantages of using these AR techniques in Virtual Museums, we verified the main forms of marking used to enlarge virtual objects, and we realized that there are still few studies that address Virtual Museums with AR Techniques aimed at teaching.
\end{abstract}

Resumo. A Realidade Aumentada (RA) apresenta comportamentos altamente adaptativos e evolutivos e grande potencial no patrimônio cultural por gerar ambientes mais atraentes e imersivos nos aspectos audiovisuais. Nesse sentido, este trabalho realiza um estudo secundário na forma de Mapeamento Sistemático, que identifica e analisa estudos primários sobre propostas de Museus Virtuais desenvolvidos utilizando técnicas de RA para educação. Levantamos algumas das vantagens da utilização dessas técnicas de RA em Museus Virtuais, verificamos as principais formas de marcação utilizadas para ampliar os objetos virtuais, e percebemos que ainda são poucos os estudos que abordam Museus Virtuais com Técnicas de RA voltadas para o ensino.

\section{Introdução}

A Realidade Virtual (RV) pode ser definida pelo uso de computadores e interfaces com o usuário para criar o efeito de mundos tridimensionais que incluem objetos interativos com uma forte sensação de presença tridimensional. De forma prática, "são realidades 
diferentes, alternativas, criadas artificialmente, mas são percebidas pelos nossos sistemas sensórios da mesma forma que o mundo físico à nossa volta" [TORI and HOUNSELL, 2018].

$\mathrm{Na}$ área de RV, durante muito tempo, real e virtual eram tratados como mutuamente exclusivos. O objetivo da RV era tirar do usuário a percepção do mundo real e fazê-lo se sentir apenas no ambiente virtual, como continua a ser hoje. Na década de 1990, no entanto, surgiu o conceito de Realidade Aumentada (RA) e a mistura entre real e virtual passou a ser uma possibilidade.

A RA, enriquece o ambiente físico com objetos sintetizados computacionalmente, permitindo a coexistência de objetos reais e virtuais. A RA mantém referências para o entorno real, transportando elementos virtuais para o espaço do usuário. O objetivo é que o usuário possa interagir com o mundo e os elementos virtuais, de maneira mais natural e intuitiva, sem necessidade de treinamento ou adaptação " [TORI and HOUNSELL, 2018].

Os avanços da Tecnologia de Informação e Comunicação (TIC), incluindo hardware e software, principalmente em dispositivos móveis, vem possibilitando que aplicações de RA sejam desenvolvidas de forma mais simples e rápida para dispositivos comuns e mais acessíveis ao público em geral.

O termo 'Museu Virtual' apareceu pela primeira vez em 1991 na, ICHIM ${ }^{1}$, uma conferência internacional sobre o uso de hipermídia e interatividade em museus. $\mathrm{O}$ termo se referia a um protótipo de museu virtual, que recebeu esse nome devido ao seu acesso ser feito remotamente via internet [HENRIQUES, 2018]. Museus virtuais têm sido utilizado em vários domínios, como por exemplo, educação [NISIOTIS, et al., 2019), lazer [DARZENTAS, et al., 2018], arte [HÜRST, et al., 2016], história [KYRIAKOU and HERMON, 2019] e muitos outros.

Por apresentarem comportamentos altamente adaptativos e evolutivos, o uso de técnicas de RA/RV apresentaram grande potencial no que envolve heranças culturais, por gerar ambientes mais atrativos e imersivos em quesitos audiovisuais [KARADIMAS, 2019]. Com estes benefícios, a procura pela virtualização de artefatos e monumentos cresceu de forma considerável.

Neste sentido, este trabalho realiza um estudo secundário na forma de mapeamento sistemático, que identifica e analisa estudos primários que evidenciam propostas de museus virtuais, desenvolvidos utilizando técnicas de RA, no contexto da educação.

\footnotetext{
${ }^{1}$ ICHIM : International Cultural Heritage Informatics Meetings
} 
O artigo está estruturado da seguinte forma: a seção 2 apresenta o protocolo do Mapeamento Sistemático; a seção 3 mostra como foi feita a Extração dos Dados; a seção 4 expõe a Síntese dos Dados; e a seção 5 aponta as Considerações Finais.

\section{Protocolo de Mapeamento Sistemático}

Este estudo foi realizado por meio de uma revisão sistemática da literatura com base na definição de estudo sistemático proposta por Fabbri (2017), onde divide o processo em três fases principais: planejamento, condução e publicação de resultados (Figura 1).

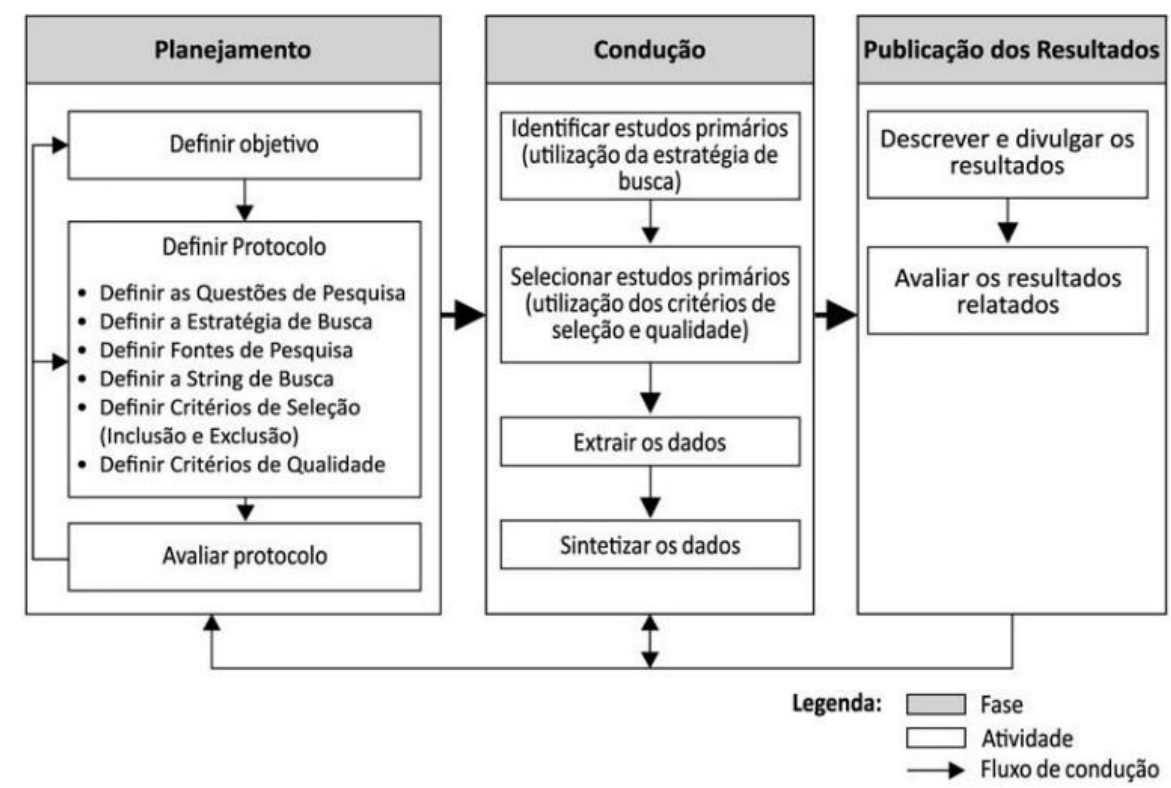

Figura 1. Fases e atividades do processo de RS [Fabbri, 2017]

O planejamento é a etapa na qual se identifica a motivação para a realização do estudo e é feita a construção do protocolo que contém as especificações das questões de pesquisa, estratégia de busca, fontes de busca e os critérios de inclusão e exclusão de estudos.

$\mathrm{Na}$ condução os estudos são identificados e selecionados de acordo com o protocolo definido na fase anterior, aplicando a estratégia de busca e os critérios de inclusão e exclusão para se encontrar todos os estudos primários disponíveis sobre o tema da pesquisa. Após a seleção dos estudos, todos os dados que interessam à pesquisa são extraídos e sintetizados por meio de um formulário de extração de dados.

$\mathrm{Na}$ publicação de resultados é realizada a escrita dos resultados do próprio Mapeamento Sistemático. Assim como uma Revisão Sistemática (RS), um Mapeamento Sistemático (MS) tem como objetivo identificar, avaliar e classificar todas as pesquisas disponíveis sobre uma determinada área de conhecimento ou tema de interesse [KITCHENHAM, CHARTERS, 2007], porém o MS visa prover uma visão mais ampla sobre o tópico da pesquisa a fim de ajudar a identificar lacunas e subtópicos para a realização de mais estudos primários [FABBRI, 2017]. 


\subsection{Objetivo e Questões de Pesquisa}

O principal objetivo deste mapeamento sistemático é identificar propostas de Museus Virtuais desenvolvidos utilizando técnicas de RA voltados para a educação. As questões de pesquisa(QP) que desejamos responder, a fim de atender esse objetivo, são:

QP1. Quais vantagens e desvantagens de usar técnicas de RA em museus virtuais para o ensino?

QP2. Qual o estado da arte de RA com museu virtual?

QP3. Quais as técnicas de marcação usadas para ampliar os objetos virtuais?

QP4. Existem relatos de uso de Museu virtual para ensino e aprendizagem?

\subsection{Estratégia de Busca (String de busca)}

Neste Mapeamento Sistemático, a seguinte string de busca foi selecionada com o intuito de receber uma quantidade maior de resultados para assim, ter uma visão mais ampla do tópico da pesquisa:

\section{"Virtual Museum" AND “Augmented Reality"}

Como resultado inicial foram encontrados 165 artigos, distribuídos em quatro bases bibliográficas digitais sendo: ACM Digital Library com 33 artigos (20\%), IEEE com 57 artigos (34,5\%), ScienceDirect com 36 artigos (21,8\%) e Scopus com 39 artigos $(23,6 \%)$.

Para auxiliar no gerenciamento e organização desde a busca até a extração de dados dos artigos selecionados, utilizamos a ferramenta virtual Parsif.al ${ }^{2}$.

\subsection{Identificação e Seleção dos Estudos Primários}

Esta seção descreve o método utilizado para selecionar os estudos relevantes para responder às questões de pesquisa deste MS. Todos os 165 artigos foram analisados e categorizados de acordo com os seguintes critérios.

\subsubsection{Critérios de inclusão}

Como se trata de um mapeamento inicial sobre o tópico de pesquisa, apenas um critério, mais abrangente, de inclusão foi escolhido para os estudos incluídos.

1. É sobre Museu Virtual desenvolvidos com técnicas de RA

\footnotetext{
${ }^{2}$ link parsif.al:

https://parsif.al//uanmacedo/systematic-review-of-virtual-museum-in-augmented-reality/
} 


\subsubsection{Critérios de exclusão}

Foram escolhidos seis critérios de exclusão para categorizar os estudos que não se encaixam na pesquisa.

1. Não é publicado como artigo completo

2. Não é um estudo primário

3. Não fala sobre Museu Virtual

4. Não fala sobre Realidade Aumentada

5. Não fala sobre Museu Virtual e Realidade Aumentada

6. Texto completo não disponível

Para um estudo ser selecionado, ele não pode se encaixar em nenhum dos critérios de exclusão. Caso ele se encaixe em ao menos um critério de exclusão, ele será categorizado e excluído da pesquisa. Caso contrário ele será selecionado para a extração dos dados.

Como mostrado na Figura 2, após realizar a busca automática nas bases de dados, 165 artigos foram identificados e 8 foram eliminados por serem duplicatas, sobrando então 157 artigos para a etapa de seleção. O processo de seleção aconteceu em duas etapas, na primeira os critérios de inclusão e exclusão foram aplicados apenas na leitura do título, resumo e palavras-chaves, gerando assim a eliminação de 92 artigos, restando então 65 artigos incluídos. Na segunda etapa os critérios foram aplicados na leitura completa dos textos resultando na eliminação de mais 21 artigos.

A partir dos 157 artigos não duplicados, 45 (28,7\%) foram excluídos por não tratarem sobre museu virtual, $19(12,1 \%)$ por não abordarem sobre Realidade Aumentada, 31(19,7\%) por não falarem nem sobre museu virtual e nem sobre Realidade Aumentada, $4(2,5 \%)$ não são artigos completos, $13(8,3 \%)$ por não serem estudos primários e apenas $1(0,6 \%)$ estudo que não teve seu texto completo encontrado.

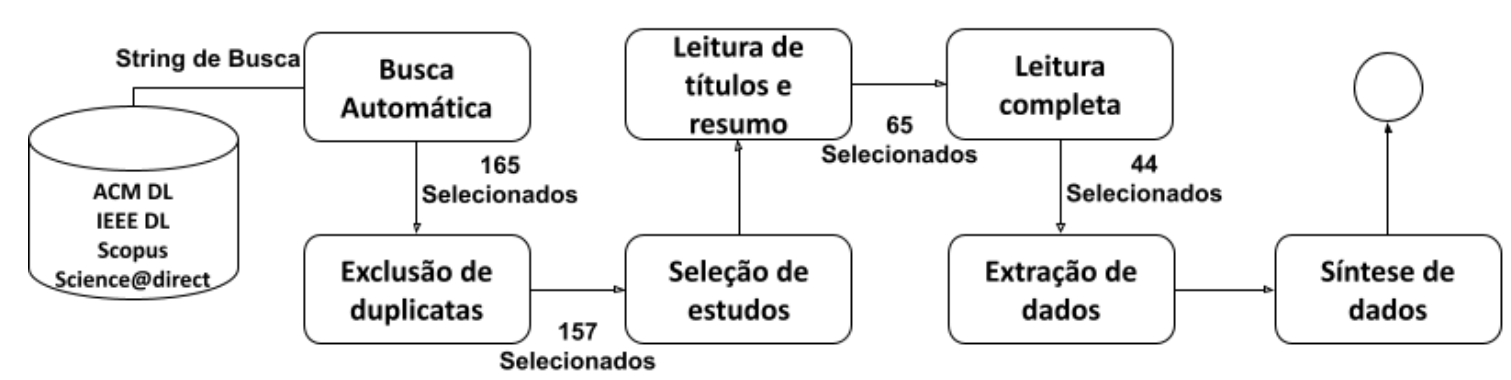

Figura 2. Atividades da fase de condução do mapeamento sistemático 


\section{Extração dos Dados}

$\mathrm{Na}$ extração de dados, foi feita a leitura completa dos 44 artigos considerados relevantes para essa pesquisa e foram marcados como incluídos na etapa de seleção. A extração dos dados buscou responder às seguintes perguntas:

1. Tem foco no ensino? (Sim/não)

2. Qual tipo de ensino?

3. Qual técnica de marcação foi usada?

4. Faz modelagem de um museu real? (sim/não)

5. Qual o domínio do museu?

6. Ferramenta usada para modelagem

7. Se for nova, qual?

8. O museu é interativo? (Sim/não)

9. Qual o tipo de pesquisa?

10. Tipo de equipamento usado para visualização

Respondendo às questões 1 e 2 , ao final da extração de dados foram encontrados $19(43,2 \%)$ artigos com foco no ensino, que tratavam desde tecnologias e métodos até Arte e História. Os outros 25 (56,8\%) não tratavam de nenhuma forma de ensino.

Com relação à questão 3, foram identificadas três técnicas de marcação: marcação por âncora, em 21 (47,7\%) artigos, sem marcação em 14 (31,8\%) artigos, marcação por localização em $5(11,3 \%)$ artigos e $4(9,1 \%)$ artigos que não citavam a técnica de marcação utilizada.

Referente às questões 4 e 5 em conjunto, também foram identificados 23 $(52,3 \%)$ artigos que estudam a virtualização de um museu real, como por exemplo a virtualização da Biblioteca de Pittsburgh [DEYOUNG and HSIEH, 2019] e 21 (47,7\%) artigos que tratam museus que foram construídos para serem virtuais, tais como o museu arqueológico na Malásia [THWAITES, et al., 2019].

Respondendo à questão 6 e 7, no que se refere às ferramentas de modelagens utilizadas, foram identificados $17(38,6 \%)$ artigos que fazem o uso do Unity3D, 4 (9.1\%) artigos utilizam o Blender, 8 (18.2\%) artigos fazem a apresentação de uma nova ferramenta de modelagem, tal como M.A.G.E.S. Plataform [GERONIKOLAKIS, et al., 2020] e $15(34,1 \%)$ estudos não citam qual ferramenta de modelagem utilizaram.

Relacionado a interatividade, questionado na pergunta 8, $30(68,2 \%)$ artigos apresentavam museus que possuem conteúdos interativos, seja por meio da audição ou de simulação do tato. Outros $13(29,5 \%)$ artigos apresentavam museus que não utilizam de uma interatividade melhorada por meios virtuais e apenas $1(2,3 \%)$ artigo não citava o seu uso. 
Para responder à questão 9, no que se refere ao tipo de pesquisa, consideramos os seis tipos definidos por Wieringa et al. (2005):

- Artigo de experiência: são estudos que relatam como algo foi feito na prática.

- Artigo de opinião: apresentam a opinião do pesquisador sobre o tópico de pesquisa.

- Artigo filosófico: refere-se a estudos que discutem uma nova estrutura para um campo de conhecimento.

- Pesquisa de avaliação: quando um estudo avalia uma solução que já está presente na indústria.

- Pesquisa de validação: quando um estudo valida uma nova solução que não foi implantada na indústria.

- Proposta de solução: são estudos que apresentam novas soluções.

Para realizar a classificação dos estudos, foi aplicada a tabela de decisão proposta por Petersen et al. (2015) mostrada na Figura 3.

\begin{tabular}{|c|c|c|c|c|c|c|}
\hline & R1 & R2 & R3 & R4 & R5 & R6 \\
\hline \multicolumn{7}{|l|}{ Condições } \\
\hline Usado na prática & V & - & v & F & $\mathrm{F}$ & $\mathrm{F}$ \\
\hline Nova solução & . & V & F & - & $\mathrm{F}$ & $\mathrm{F}$ \\
\hline Avaliação empírica & $\mathrm{V}$ & $\mathrm{F}$ & F & $\mathrm{V}$ & $\mathrm{F}$ & $\mathrm{F}$ \\
\hline Estrutura conceitual & . & . & - & . & $\mathrm{V}$ & $\mathrm{V}$ \\
\hline Opinião sobre algo & $\mathrm{F}$ & $\mathrm{F}$ & $\mathrm{F}$ & F & $\mathrm{F}$ & $\mathrm{V}$ \\
\hline Experiência do autor & . & . & - & . & $\mathrm{F}$ & $\mathrm{F}$ \\
\hline \multicolumn{7}{|l|}{ Decisões } \\
\hline Pesquisa de avaliação & $\checkmark$ & . & - & . & . & . \\
\hline Proposta de solução & - & $\checkmark$ & - & - & - & \\
\hline Pesquisa de validação & . & . & . & $\checkmark$ & $\cdot$ & . \\
\hline Artigos filosóficos & . & . & . & . & $\checkmark$ & . \\
\hline Artigos de opinião & - & - & - & - & - & V \\
\hline Relato de experiência & . & - & $\sqrt{ }$ & . & - & . \\
\hline
\end{tabular}

Figura 3. Fonte: traduzido de (PETERSEN et al., 2015)

Legenda: $\mathrm{R} 1$ - $\mathrm{R} 6$ = tipo de Pesquisa; $\mathrm{V}=$ Verdadeiro; $\mathrm{F}=$ Falso; $\bullet=$ irrelevante ou não aplicado; R1 - R6 = referente às regras aplicadas.

E concluímos que $25(56,8 \%)$ estudos apresentam Pesquisa de validação, 12 (27,3\%) Pesquisa de avaliação, 6 (13,6\%) Proposta de solução e 1 (2,3\%) Artigo de opinião. Com esses dados, podemos observar que apesar de existirem muitos estudos de caso, muitos deles ainda estão na fase de prototipagem ou experimentos fechados. 
Respondendo a pergunta 10 , no que se refere a equipamentos de visualização, foram encontrados $23(52,3 \%)$ estudos que utilizavam dispositivos 'head-mounted', que são dispositivos que precisam ser usados na cabeça do usuário, como o HoloLens(WANG, 2020), 20(45,4\%) estudos que utilizam visualização por dispositivos móveis e apenas $1(2,3 \%)$ artigo que não cita seu meio de visualização.

\section{Síntese dos dados}

Uma vez os dados extraídos dos estudos selecionados como relevantes para esse Mapeamento Sistemático, foi realizada uma síntese dos dados com o objetivo de responder às questões de pesquisa propostas neste estudo.

4.1. Sobre a Questão de Pesquisa 1: Quais vantagens e desvantagens de usar técnicas de RA em museus virtuais para o ensino?

Após a extração dos dados foram identificados 30 (68,2\%) estudos de Museus Virtuais que demonstram que um dos principais motivadores para a utilização de tecnologias de RA em Museus Virtuais é a maior imersão e interação que os tornam muito atrativos. Outro benefício gerado pelo surgimento de Museus Virtuais é a preservação de artefatos, pois, a maioria dos artefatos disponíveis em museus ficam protegidos por vidros ou distante do público para que não ocorram acidentes ou roubos. A RA proporciona uma interação maior com áudios, textos e imagens sobre uma determinada amostra sem arriscar a peça original, e também provê também maior portabilidade, tendo em vista que para mover um objeto de um museu para outro, não há a necessidade de transportar o objeto real e sim, apenas seu modelo virtual.

\subsection{Sobre a Questão de Pesquisa 2: Qual o estado da arte de RA com Museu} Virtual?

Ao se pesquisar sobre Realidade Aumentada com Museu Virtual, percebe-se que existem poucos estudos que abordam o ensino, mas ainda assim eles abrangem vários temas, que vão desde novos frameworks de modelagem 3D até a criação de novos métodos de apresentação e guia dentro de museus para gerar maior engajamento entre os visitantes.

Os domínios encontrados percorrem todo o globo, apresentando, por exemplo, uma reconstrução de igrejas na Itália [BANFI, et al., 2019] ou um museu virtual de arte japonês [LIN, 2018]. Identificamos $23 \quad(52,3 \%)$ estudos que fazem a modelagem de museus reais e 21 (47.7\%), que criam museus novos para os seus próprios temas.

Em relação a novas soluções, foram classificados 6 (13.6\%) artigos como propostas de soluções, que apresentam uma nova ferramenta de modelagem 
[RATTANARUNGROT, 2016] e novas arquiteturas e passos para o mapeamento 3D [HU, Q., 2017].

4.3. Sobre a Questão de Pesquisa 3: Quais as técnicas de marcação usadas para ampliar os objetos virtuais?

Ao se estudar sobre Realidade Aumentada, encontra-se três técnicas para ampliar objetos virtuais, Realidade aumentada com marcador, Realidade Aumentada sem marcador e Realidade Aumentada baseada em localização.

\subsubsection{Realidade Aumentada com marcador}

Foram identificados $21(47,7 \%)$ artigos que utilizam este tipo de marcação. Utiliza a tecnologia de reconhecimento de imagem para identificar algum tipo de marcador visual, como um Código QR por exemplo [S. PUSPASARI, 2019], para reproduzir o objeto virtual que está relacionado a este marcador. Os marcadores podem ser de qualquer tipo, contanto que tenham padrões distintos para que o reconhecimento seja realizado de maneira rápida e simples.

\subsubsection{Realidade Aumentada sem marcador}

Foram identificados 14 (31,8\%) artigos que utilizam este tipo de marcação. Também é chamada de Realidade Aumentada baseada em projeção, faz-se a projeção de seus objetos virtuais com base em superfícies no mundo real, sendo assim não depende de algum ponto pré-determinado ou alguma âncora específica para o objeto.

\subsubsection{Realidade Aumentada baseada em localização}

Foram identificados $5(11,3 \%)$ artigos que utilizam este tipo de marcação. Utilizando as tecnologias de GPS, bússola digital, medidor de velocidade e outras aplicações incorporadas ao dispositivo, este tipo de projeção se baseia em coordenadas geográficas para ampliar seus objetos sendo bem comuns em aplicações de dispositivos móveis, como mapas e jogos.

4.4. Sobre a Questão de Pesquisa 4: Existem relatos de uso de Museu virtual para ensino e aprendizagem?

Ao final da extração de dados, foram identificados 19 (43,2\%) estudos que utilizavam a Realidade Aumentada em Museus Virtuais para algum tipo de ensino, em maioria tratando sobre alguma tecnologia e suas aplicações e efeitos em Museus Virtuais.

\section{Considerações Finais}

Um dos principais problemas enfrentados na realização de um estudo sistemático é a sua alta complexidade, principalmente para pesquisadores iniciantes que não possuem experiência necessária para encontrar e selecionar estudos adequados para sua pesquisa. Para contornar essa dificuldade, o mapeamento sistemático possui um protocolo que é 
elaborado com a ajuda de outros pesquisadores mais experientes, tanto no tema da pesquisa quanto em estudos sistemáticos.

Para auxiliar na condução deste mapeamento sistemático, foi feito o uso de uma ferramenta para busca automática e organização de estudos e um acompanhamento sobre as decisões de classificação do pesquisador principal.

Identificamos que os principais motivadores para a utilização de tecnologias de RA/RV em Museus Virtuais é a maior imersão e interação, que os tornam muito atrativos, e a preservação dos artefatos. Mas, ainda existem poucos trabalhos que abordam o tema, principalmente voltados ao ensino.

Também identificamos uma maior parcela do uso de dispositivos móveis e projeção por marcação nos projetos, o que torna a virtualização mais barata e simples de se realizar. Apesar disto, acredita-se que o uso de dispositivos Head-Mounted, como o microsoft-lens por exemplo, podem tornar o projeto muito mais atrativo para o público, principalmente por sua diferença física de não haver a necessidade de segurar um objeto nas mãos e sim um óculos no rosto, o que pode gerar uma imersão e interatividade muito maior.

\section{Referências}

Banfi, F., Brumana, R., and Stanga, C. (2019). Extended reality and informative models for the architectural heritage: from scan-to-BIM process to virtual and augmented reality.

Brereton, P., Kitchenham, B. A., Budgen, D., Turner, M., and Khalil, M. (2007). Lessons from applying the systematic literature review process within the software engineering domain. Journal of systems and software, 80(4), 571-583.

DeYoung, J., \& Hsieh, A. H. H. (2019, June). Augmentations in the Palace of Culture: Animate Soundscapes at the Carnegie Library of Pittsburgh. In 2019 IEEE Games, Entertainment, Media Conference (GEM) (pp. 1-5). IEEE.

Geronikolakis, E., Zikas, P., Kateros, S., Lydatakis, N., Georgiou, S., Kentros, M., \& Papagiannakis, G. (2020). A True AR Authoring Tool for Interactive Virtual Museums.

Henriques, R. M. N. (2018). Os museus virtuais: conceito e configurações.

Hürst, W. C., \& De Coninck, F. F. \& Tan, XJ (2016). Complementing Artworks to Create Immersive VR Museum Experiences. In Proceedings of the 13th International Conference on Advances in Computer Entertainment Technology (Vol. 34, pp. 1-6).

Kitchenham, B., and Charters, S. (2007). Guidelines for performing systematic literature reviews in software engineering.

Kyriakou, P., \& Hermon, S. (2019). Can I touch this? Using natural interaction in a museum augmented reality system. Digital Applications in Archaeology and Cultural Heritage, 12, e00088. 
Lin, T. G., Shih, H. L., Lee, C. T., Hsieh, H. Y., Chen, Y. Y., and Liu, C. K. (2018). Omni-learning XR technologies and Visitor-centered Experience in the Smart Art Museum. In 2018 IEEE International Conference on Artificial Intelligence and Virtual Reality (AIVR) (pp. 258-261). IEEE.

Nakagawa, E. Y., Scannavino, K. R. F., Fabbri, S. C. P. F., and Ferrari, F. C. (2017). Revisão sistemática da literatura em engenharia de software: teoria e prática.

Nisiotis, L., Alboul, L., and Beer, M. (2019). Virtual Museums as a New Type of Cyber-Physical-Social System. In International Conference on Augmented Reality, Virtual Reality and Computer Graphics (pp. 256-263). Springer, Cham.

Petersen, K., Vakkalanka, S., and Kuzniarz, L. (2015). Guidelines for conducting systematic mapping studies in software engineering: An update. Information and Software Technology, 64, 1-18.

Rattanarungrot, S., and White, M. (2016, October). Development of service oriented mobile AR applications for museum learning activities. In 2016 22nd International Conference on Virtual System \& Multimedia (VSMM) (pp. 1-8). IEEE.

Thomas, P. C., and David, W. M. (1992, January). Augmented reality: An application of heads-up display technology to manual manufacturing processes. In Hawaii international conference on system sciences (pp. 659-669).

Thwaites, H., Santano, D., Esmaeili, H., \& See, Z. S. (2019). A Malaysian cultural heritage digital compendium. Digital Applications in Archaeology and Cultural Heritage, 15, e00116.

Tori, R. and Hounsell,M. S.(Eds.). (2018). Introdução a Realidade Virtual e Aumentada. Porto Alegre:Editora SBC

Wang, G., et al. (2020) Application of Mixed Reality Technology in Education with the case of a Huangmei Opera Cultural Education System. In: 2020 IEEE 2nd International Conference on Computer Science and Educational Informatization (CSEI). p. 301-305.

Wieringa, R., Maiden, N., Mead, N., and Rolland, C. (2006). Requirements engineering paper classification and evaluation criteria: a proposal and a discussion. Requirements engineering, 11(1), 102-107.

\section{Estudos selecionados}

Nesta seção iremos incluir uma lista de todos os estudos encontrados que foram aprovados pelos critérios de inclusão e exclusão.

https://docs.google.com/document/d/1aALA0HkB0RsksbQRgxNtW44ytEQk4oMITJmj TDJisfc/edit?usp=sharing 